\title{
Half-Quantum Vortices and other Mysteries in an Unconventional Superconductor
}

Observation of half-height magnetization steps in $\mathrm{Sr}_{2} \mathrm{RuO}_{4}$

Authors: J. Jang, D.G. Ferguson, V. Vakaryuk, R. Budakian, S.B. Chung, P.M. Goldbart and Y. Maeno

Science 331, 186 (January 14, 2011); arXiv:1101.3611

\section{Recommended and a Commentary by Manfred Sigrist, ETH Zurich, Switzerland}

Introduction: Shortly after its discovery $\mathrm{Sr}_{2} \mathrm{RuO}_{4}$ was declared an exemplary case of a spin-triplet superconductor and an electronic analog of superfluid ${ }^{3} \mathrm{He}$. While there is evidence supporting the realization of a chiral $p$-wave pairing state (analog to the A-phase of ${ }^{3} \mathrm{He}$ ), there have been also numerous findings which are not so simply understood [1]. These include line(s) of zero in the gap necessary to understand the low-temperature power law observed in specific heat, heat conductance, London penetration depth etc., which appear incompatible with chiral p-wave pairing of the generic form,

$$
\hat{\Delta}_{\vec{k}}=i \vec{d}(\vec{k}) \cdot \hat{\vec{\sigma}} \hat{\sigma}^{y} \quad \text { with } \quad \vec{d}(\vec{k})=\Delta_{0} \hat{z}\left(k_{x} \pm i k_{y}\right) .
$$

in which the gap function corresponds to an equal-spin pairing state (spin orientation perpendicular to $z$-axis) with an orbital angular momentum $\hat{l}$ along the $z$-axis. The corresponding quasi-particle gap $\left|\Delta_{\vec{k}}\right|=|\vec{d}(\vec{k})|$ has no zeroes.

Eq.(1) assumes that spin-orbit coupling pins the $\vec{d}$-vector orientation to lie along the $z$-axis of the tetragonal crystal, compatible with the early observation of a constant NMR- Knight shift for fields in the basal plane [2]. But soon no change of the Knight shift was found for fields along the $z$-axis too [3]. This was interpreted as a field-induced flip of the $\vec{d}$-vector orientation, which should occur for fields even smaller than 200 Oe and indicates that the anisotropy energy for the $\vec{d}$-vector is rather small. Indeed without spin-orbit coupling there would be six degenerate odd-parity states within weak-coupling theory, among which four are equal-spin pairing state with spin orientation parallel to $\hat{z}(\vec{d} \perp \hat{z})$. Therefore, it is not inconceivable that the critical field to turn the $\vec{d} \perp \hat{z}$ is not large.

Half-flux quantization: For chiral p-wave pairing with the $\vec{d}$-vector in the basal plane and low anisotropy for it within the basal plane, it was speculated that vortices with half-flux quanta $\Phi_{0} / 2$ could occur in the appropriate geometries for fields along the $z$-axis. This has been of particular interest since such vortices contain zero-energy quasi-particles states with Majorana Fermion character, interesting for topological quantum computing [5]. Now a remarkable new experiment, highlighted above, reports the observation of "half-height magnetization steps" in mesoscopic torus-shaped samples of $\mathrm{Sr}_{2} \mathrm{RuO}_{4}$ which are interpreted as a half-flux-quantum vortex [4]. Aspects of it are not well understood. There is also the need for a theory for the energetics of such topological structures in a state compatible with line of zeroes mentioned above.

The axis of the hole in the torus is along the $z$-axis. The measurement use a recently developed cantilever magnetometer. Under usual conditions, with a magnetic field along the $z$-axis, standard flux quantization steps are observed in the flux threading the torus hole. However, if an additional 
magnetic field is applied in the basal plane a narrow window opens up for the $z$-axis field where an intermediate magnetization step enters, corresponding most likely to half of a flux quantum $\Phi_{0}$. A $z$ axis field sweep covers the whole sequence of steps, $n=-2,-3 / 2,-1,-1 / 2,0,+1 / 2,+1,+3 / 2,+2$. This behavior is present for arbitrary orientations of the field within the basal plane and the threshold field necessary to see the new step feature is as low as roughly 10 Oe.

How should we interpret this finding? And what is the role of the in-plane field which seems to act as a kind of catalyst? Understanding the experiment and the explanation favored by the authors needs a brief introduction to the idea of fractional vortices:

Fractionally quantized vortices: Such vortices date back to discussions of superfluid ${ }^{3} \mathrm{He}[7,8]$. Generally spin-triplet order parameters allow for both orbital and spin contributions (rotations of the $\vec{d}$-vector) to the phase change of the order parameter. Unusual flux quantization appears in superconductors, when the two are combined keeping the order parameter single-valued. In particular, a half-flux quantum is realized, if the spin and the orbital part each contribute $\pi$ to the overall phase winding; only the orbital part contributes to the magnetic flux, the spin part yields spin currents which are more difficult to observe. Recently the stability of this kind halfflux vortices in various geometries $[6,9]$ has been examined. One major obstacle in the energetics lies in the spin currents induced in such flux line structures, which remain unscreened (unlike the charge currents). They would spread throughout the whole sample and lead to a 'divergent' (volume dependent) energy. Therefore, it was suggested that half-flux lines could exist only in rather small samples, a condition likely met by the present experiment. Additionally a small spin superfluid density relative to the orbital (charge) part would be favorable to stabilize a half-flux vortex [6]. Vakaryuk and Leggett showed that Fermi liquid corrections work in this direction and, moreover that, rotations of the $\vec{d}$-together with orbital currents would be accompanied by a spin magnetization whose orientation depends on the orientation of $\vec{d}[9]$. Ideas and calculations on further modifying the energetics of such topological defects involve the idea of a soliton-like flip of the d-vector ("d-soliton") which take the aspect of spin-orbit coupling into account [10].

In Fig.1 I show several situations for the superconducting torus. The left panel displays the standard case of integer flux quantization, $\Phi=\Phi_{0} n$. The center panel the one with the d-soliton (red) which acts like a " $\pi$-link" (sign change of the $\vec{d}$-vector), such that the flux quantization follows a halfinteger rule $\Phi=\Phi_{0}(n+1 / 2)$. The $\vec{d}$-soliton costs energy, analogous to the above mentioned d-vector textures inducing spin currents. This soliton energy would be proportional to the area of the soliton wall. Thus, the energy has to be balanced the coupling of the flux to applied $z$-axis magnetic field. However, this may not be sufficient and further modifications involving structure in the spin-density may be needed. Here the inplane field come into action. Analogous to the situation described by Vakaryuk and Leggett the d-soliton generate a spin density (inplane, parallel to the soliton wall, see Fig. 1 right panel) which couples to the inplane magnetic field and lowers the energy additionally. This may indeed be the "catalytic" role of the inplane magnetic field in this experiment. 


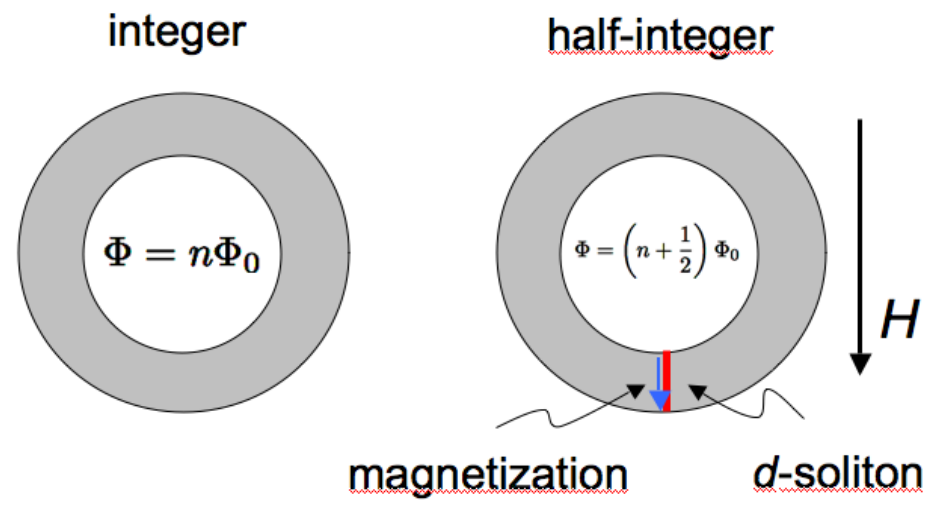

Fig.1: Flux quantization in the torus: Left panel: standard situation; Right panel: d-soliton connecting inside and outside of the torus yields a $\pi$-shift which lead to half-integer quantization.

Note the inplane spin magnetization is parallel to the soliton wall in our simple model.

Conclusions on the puzzling properties of $\mathrm{Sr}_{2} \mathrm{RuO}_{4}$ : The observation of half-flux vortices is compatible with the chiral $p$-wave state. It fits well with spin-triplet pairing, but cannot be counted as evidence for chirality. Actually, one of the important clues for the chiral $p$-wave state, the existence of edge currents [11], could not be confirmed within this experiment [4]. We encounter here the same (frustrating) situation as in the earlier experiments by Kirtley and collaborators $[12,17]$ who reported a negative result for their search for edge currents. It remains an open question why edge currents are possibly much smaller than expected [17]. On the other hand, the chiral $p$-wave state seems to the fit very well to the observation of the polar Kerr effect by the group of A. Kapitulnik [13]. Nevertheless, it has turned out difficult to give a quantitative account for the measured magnitude of the signal from the theoretical side $[14,15,16,17]$.

A long-standing puzzle is still the apparent limiting behavior observed in $H_{c 2}$ for inplane fields, which goes against the expectations [1]. The inplane equal-spin pairing does not show a drop of the spin susceptibility in the superconducting phase [2], such that standard paramagnetic limiting does not provide an explanation.

In summary, the experiments by Jang et al adds a new intriguing twist to the story of $\mathrm{Sr}_{2} \mathrm{RuO}_{4}$ and demonstrates the inexhaustible supply of novel physics offered by this material.

I would like to acknowledge helpful discussions with D.F. Agterberg, A. Bouhon, C. Kallin, H.Y. Kee, Y.B. Kim, Y. Maeno and T.M. Rice on this topic.

\section{References}

[1] A.P. Mackenzie and Y. Maeno, Rev. Mod. Phys. 75, 657 (2003).

[2] K. Ishida et al., Nature 396, 658 (1998).

[3] H. Murakawa et al., Phys. Rev. Lett. 93, 167004 (2004). 
[4] J. Jang, D.G. Ferguson, V. Vakaryuk, R. Budakian, S.B. Chung, P.M. Goldbart and Y. Maeno, Science 331, 186 (2011).

[5] D.A. Ivanov, Phys. Rev. Lett. 86, 268 (2001).

[6] S.B. Chung, H. Bluhm and E.A. Kim, Phys. Rev. Lett. 99, 197002 (2007).

[7] D. Vollhardt and P. Wölfle, The Superfluid Phases of Helium 3, Taylor \& Francis (1990).

[8] M.M. Salomaa and G.E. Volovik. Rev. Mod. Phys. 59, 533 (1987).

[9] V. Vakaryuk and A.J. Leggett, Phys. Rev. Lett. 103, 057003 (2009); V. Vakaryuk, Phys. Rev. Lett. 101, 167002 (2008).

[10] H.Y. Kee. Y.B. Kim and K. Maki, Phys. Rev. B62, R9275 (2000).

[11] M. Matsumoto and M. Sigrist, J. Phys. Soc. Jpn. 68, 994 (1999).

[12] J.R. Kirtley et al., Phys. Rev. B76, 014526 (2007).

[13] J. Xia et al., Phys. Rev. Lett. 97, 167002 (2006).

[14] R.M. Lutchyn, P. Nagornykh and V.M. Yakovenko, Phys. Rev. B 80, 104508 (2009).

[15] R. Roy and C. Kallin, Phys. Rev. B 77, 174513 (2008).

[16] J. Goryo, Phys. Rev. B 78, 060501(R) (2008).

[17] C. Kallin and A. J. Berlinsky, J. Phys.: Condens. Matter 21, 164210 (2009). 